\title{
Nivolumab a New Standard of Care in Adjuvant Settings in Esophageal and Gastroesophageal Junctional Cancer Patients
}

\author{
Senthil J. Rajappa ${ }^{1}$ Syed Nusrath ${ }^{2}$ \\ ${ }^{1}$ Department of Medical Oncology, Basavatarakam Indo American \\ Cancer Institute and Research Institute, Hyderabad, Telangana, India \\ 2 Department of Surgical Oncology, Division of Upper Gastrointestinal \\ Surgery, Basavatarakam Indo American Cancer Institute and \\ Research Institute, Hyderabad, Telangana, India
}

Ind J Med Paediatr Oncol 2021;42:468-469.
Address for correspondence Syed Nusrath, MS, DNB, Department of Surgical Oncology, Division of Upper Gastrointestinal Surgery, Basavatarakam Indo American Cancer Institute and Research Institute, Hyderabad, Telangana, 500034, India (e-mail: dr.nusrath2008@gmail.com).
There exists a significant risk of recurrence after neoadjuvant chemoradiotherapy (NCRT) and surgery among the threefourth of patients with carcinoma esophagus who do not achieve complete pathological remission (PCR). ${ }^{1}$ Therefore, CheckMate 577 (CM577), a global, randomized, doubleblind, placebo-controlled phase 3 trial, has explored the role of an immune checkpoint inhibitor (ICPI), Nivolumab as adjuvant therapy in patients with esophageal or gastroesophageal junction (GEJ) cancer who did not achieve a PCR. ${ }^{2}$

In clinical trials of previously treated patients with advanced esophageal or GEJ tumors with squamous-cell carcinoma (SCC) or adenocarcinoma histology, the overall survival (OS) in the nivolumab arm was higher than those who received either chemotherapy or placebo., ${ }^{3,4}$ These results led to the conduct of the CM577 study.

The authors need to be commended for carrying out such a unique study of its kind. Among patients with completely resected stage II or III esophageal or GEJ cancer (R0 resection) who had received NCRT and did not achieve a PCR, an impressive doubling of disease-free survival (DFS) was seen in the nivolumab arm in comparison with the placebo arm. However, the literature to support DFS as a valid surrogate to OS in esophageal cancer is not robust. ${ }^{5,6}$ We eagerly await to see if these results translate into an OS benefit.

We wish to highlight certain interesting observations from the publication that are worth discussing. Surgery for esophageal cancer is complex and requires substantial expertise. However, there is hardly any data in the
CM577 study regarding the adequacy and type of surgery performed, the number of fields dissected, nodal yield, proficiency of surgeons, and surgical quality control. Quality and adequacy of surgery are essential components of esophageal cancer treatment, allows for optimal staging, improves locoregional disease control, and probably survival. With inadequate surgery, the opportunity to remove diseased nodes is missed out, leading to the erroneous assignment of post-therapy nodal status, resulting in inferior outcomes. ${ }^{7}$

In the multicentric randomized NEOCRETEC 5010 trial evaluating the role of NCRT in stage II and III esophageal SCC, where the standards of surgery were high, the median DFS in surgery alone arm was 41.7 months at 34.6 months of followup. ${ }^{8}$ In CM577, at 2 years median follow-up, the median DFS was 29 months on the adjuvant Nivolumab arm for patients with SCC and R0 resection. This DFS on the CM577, despite preoperative chemoradiation, R0 resection, and adjuvant nivolumab, is grossly inferior to the DFS on the surgery alone arm of the NEOCRETEC 5010 study. One of the potential reasons for the inferior outcome in the CM577 could be the poor quality of surgery.

In the CROSS trial, the benefits of NCRT in terms of OS were better for squamous histology than adenocarcinoma. ${ }^{9}$ Hence, in the CM577 trial, we would expect the DFS on the placebo arms to be higher for squamous histology than adenocarcinoma. However, for unexplained reasons, the DFS on the placebo arms was strikingly similar for both histologies at 11 months.
DOI https://doi.org/ 10.1055/s-0041-1739189. ISSN 0971-5851. (c) 2021. Indian Society of Medical and Paediatric Oncology. All rights reserved.

This is an open access article published by Thieme under the terms of the Creative Commons Attribution-NonDerivative-NonCommercial-License, permitting copying and reproduction so long as the original work is given appropriate credit. Contents may not be used for commercial purposes, or adapted, remixed, transformed or built upon. (https://creativecommons.org/ licenses/by-nc-nd/4.0/)

Thieme Medical and Scientific Publishers Pvt. Ltd., A-12, 2nd Floor, Sector 2, Noida-201301 UP, India 
The CM577 study used a tumor proportion score (TPS) rather than a combined positive score (CPS) to quantify PDL1 expression. Adjuvant nivolumab in subset analysis did not show benefit on PDL1 expression by TPS. However, post hoc analysis by CPS showed that patients with higher CPS $(>5)$ benefitted from adjuvant nivolumab (29.4 vs. 10.2 months). In those with CPS $<5(n=295)$, the difference was smaller (16.3 vs. 11.1 months), hazard ratio (HR) was 0.89 , and confidence interval $(\mathrm{CI})$ was $0.65-1.22$.

In recently published ICPI studies in advanced esophageal/GEJ and gastric cancers, the PDL1 expression only by CPS correlated with outcomes. In the Checkmate 649 and Keynote 590 trials, patients whose tumors had higher CPS benefitted better with the addition of ICPI to chemotherapy. ${ }^{10,11}$ In the subset analysis of the Keynote 590 study, the OS benefit was driven by the subset of patients with higher CPS $(>10) .{ }^{11}$ Similarly, in Checkmate 649, the clinical significance of OS benefit of 2 months with an HR of $0.8(0.68-0.94, p=0002)$ in the intention to treat (ITT) population is questionable. ${ }^{10}$ We understand that PDL1 is not a robust biomarker. The magnitude of nivolumab benefit may be better in patients with a higher PDL1 expression by CPS based on CM577 and other ICPI studies in advanced settings. We feel that it would be appropriate to stratify patients in CM577 based on CPS rather than TPS.

In the subset analysis, DFS was better when patients were randomized $\geq 10$ weeks after surgery compared with those randomized earlier. Earlier institution of adjuvant systemic therapy is associated with better outcomes than delayed therapy. A common reason for the delayed start of adjuvant therapy is delayed recovery from surgery, which could reflect the need for more extensive surgery, and is associated with higher disease stages, poor biology, and inferior outcomes. This finding in CM577 is contrary to our expectations and is worth further analysis.

The absolute magnitude of DFS benefit of adjuvant nivolumab was better for SCC (29.7 vs. 11 months) than adenocarcinoma (19 vs. 11 months). Similarly, whether the lack of benefit in GEJ tumors could be due to a higher proportion of adenocarcinoma in this population, is worth a thought. This differential effect of adjuvant nivolumab based on the histology and anatomical subsite needs further evaluation. In the forest plot, patients with ypT3T4 seemed to have a better outcome in the standard arm compared with lower stages, which is not expected. This is possible only if the lower $\mathrm{T}$ stages had more patients with nodal positivity. However, this information is missing in the study.

Although our observations are based on subset analysis and hypotheses generation, these findings are quite glaring and clinically relevant to be ignored. We feel that the benefits of adjuvant nivolumab in terms of DFS are likely to be restricted to patients with esophageal SCC. In the absence of a demonstrable OS benefit so far, clinicians need to be judicious while recommending adjuvant nivolumab to all subsets of patients as approved by the Foods and Drugs Administration (FDA).

\section{Declaration}

The authors disclose no financial and personal relationships with other people or organizations that could inappropriately influence (bias) their work.

\section{Funding \\ None.}

\section{Conflict of Interest}

None declared.

\section{References}

1 Blum Murphy M, Xiao L, Patel VR, et al. Pathological complete response in patients with esophageal cancer after the trimodality approach: the association with baseline variables and survivalThe University of Texas MD Anderson Cancer Center experience. Cancer 2017;123(21):4106-4113

2 Kelly RJ, Ajani JA, Kuzdzal J, et al; CheckMate 577 Investigators. Adjuvant nivolumab in resected esophageal or gastroesophageal junction cancer. N Engl J Med 2021;384(13):1191-1203

3 Kang Y-K, Boku N, Satoh T, et al. Nivolumab in patients with advanced gastric or gastro-oesophageal junction cancer refractory to, or intolerant of, at least two previous chemotherapy regimens (ONO-4538-12, ATTRACTION-2): a randomised, double-blind, placebo-controlled, phase 3 trial. Lancet 2017;390 (10111):2461-2471

4 Kato K, Cho BC, Takahashi M, et al. Nivolumab versus chemotherapy in patients with advanced oesophageal squamous cell carcinoma refractory or intolerant to previous chemotherapy (ATTRACTION-3): a multicentre, randomised, open-label, phase 3 trial. Lancet Oncol 2019;20(11):1506-1517

5 Petrelli F, Tomasello G, Barni S. Surrogate end-points for overall survival in 22 neoadjuvant trials of gastro-oesophageal cancers. Eur J Cancer 2017;76:8-16

6 Oba K, Paoletti X, Alberts S, et al; GASTRIC group. Disease-free survival as a surrogate for overall survival in adjuvant trials of gastric cancer: a meta-analysis. J Natl Cancer Inst 2013;105(21): $1600-1607$

7 Nusrath S, Saxena AR, Raju KVVN, Patnaik S, Subramanyeshwar Rao T, Bollineni N. The Value of Lymphadenectomy Post-Neoadjuvant Therapy in Carcinoma Esophagus: a Review. Indian J Surg Oncol 2020;11(03):538-548

8 Yang H, Liu H, Chen Y, et al; AME Thoracic Surgery Collaborative Group. Neoadjuvant Chemoradiotherapy Followed by Surgery Versus Surgery Alone for Locally Advanced Squamous Cell Carcinoma of the Esophagus (NEOCRTEC5010): a phase III multicenter, randomized, open-label clinical trial. J Clin Oncol 2018;36(27): 2796-2803

9 Shapiro J, van Lanschot JJB, Hulshof MCCM, et al; CROSS study group. Neoadjuvant chemoradiotherapy plus surgery versus surgery alone for oesophageal or junctional cancer (CROSS): longterm results of a randomised controlled trial. Lancet Oncol 2015; 16(09):1090-1098

10 Moehler M, Shitara K, Garrido M, et al. Nivolumab (Nivo) plus chemotherapy (chemo) versus chemo as first-line (1L) treatment for advanced gastric cancer/gastro-esophageal junction cancer (GC/GEJC)/esophageal adenocarcinoma (EAC): first results of the CheckMate 649 study. Ann Oncol 2020;31(Suppl 4): S1142-S1215

11 Kato KS, Sun JM, Shah MA, et al. Pembrolizumab plus chemotherapy versus chemotherapy as first-line therapy in patients with advanced esophageal cancer: the phase 3 KEYNOTE-590 study. Ann Oncol 2020;31(Suppl 4):S1142-S1215 